\title{
INTEGRO-DIFFERENTIAL EQUATIONS WITH CONSTANT LIMITS OF INTEGRATION.
}

BY DR. I. A. BARNETT.

(Read before the American Mathematical Society December 28, 1918.)

Consider the linear integro-differential equation

$$
\frac{\partial}{\partial \tau} u(\xi, \tau)=\int_{a}^{b} K(\xi, \eta) u(\eta, \tau) d \eta
$$

where $\tau$ is a real variable ranging over $\left|\tau-\tau_{0}\right| \leqq c$, and $K(\xi, \eta)$ is a continuous function defined in the square $a \leqq \xi \leqq b, a \leqq \eta \leqq b$. Volterra has shown* that the most general solution of this equation, reducing for $\tau=\tau_{0}$ to the arbitrary continuous function $u_{0}(\xi)$, is given by

$$
u(\xi, \tau)=u_{0}(\xi)+\int_{a}^{b} L(\xi, \eta, \tau) u_{0}(\eta) d \eta,
$$

where

$$
L(\xi, \eta, \tau)=\sum_{n=1}^{\infty} \frac{\left(\tau-\tau_{0}\right)^{n}}{n !} K_{n}(\xi, \eta),
$$

the functions $K_{n}$ denoting, as usually, the iterated kernels of $K$ with the understanding that $K_{1}=K$.

It is proposed in this paper to give a solution of equation (1) in terms of the characteristic numbers and functions of the kernel $K$ for the cases in which $K$ is symmetric and skewsymmetric. Furthermore, some extensions of the theory to more general kernels will be pointed out and a formal analogy between integro-differential equations and partial differential equations will be shown. It is of interest to note that equation (1) may be considered as the limiting case of the finite system of differential equations with constant coefficients

$$
\frac{d u_{i}}{d \tau}=\sum_{j=1}^{n} K_{i j} u_{j} \quad(i=1, \cdots, n),
$$

and the results for the integro-differential equations could be predicted from the well-known theory of this last system. $\dagger$

* Volterra, Lincei Rendiconti, serie 5 (1914), 2d semester, p. 394.

† Schlesinger, "Zur Theorie der linearen Integrodifferentialgleichungen," Jahresbericht der deutschen Mathematiker-Vereinigung, vol. 24 (1915-16), p. 84 . 


\section{$\S 1$. The Symmetric Kernel.}

Theorem. Through the element $\left(\tau_{0}, u_{0}\right)$, where $u_{0}$ is a real continuous function of $\xi$, there exists one and but one solution of equation (1), viz.,

(3) $v(\xi, \tau)=u_{0}(\xi)+\sum_{n=1}^{\infty} \varphi_{n}(\xi)\left(e^{\left(\tau-\tau_{0}\right) / \lambda_{n}}-1\right) \int_{a}^{b} \varphi_{n}(\eta) u_{0}(\eta) d \eta$,

where the $\lambda_{n}$ are the characteristic numbers of the kernel $K$ (necessarily real) and the $\varphi_{n}$ are the characteristic functions.

The $\lambda_{n}$ are arranged, as usually, in the order of their increasing absolute values, and the $\varphi_{n}$ are supposed normalized and orthogonal.

Two methods of proof suggest themselves. One could start with Volterra's solution (2), and obtain the form (3), by making use of the well known properties concerning iterated kernels of a symmetric kernel.* A better method, however, is to proceed directly.

Consider the infinite sum

$$
S(\xi, \tau)=\sum_{n=1}^{\infty} \varphi_{n}(\xi)\left(e^{\left(\tau-\tau_{0}\right) / \lambda_{n}}-1\right) \int_{a}^{b} \varphi_{n}(\eta) u_{0}(\eta) d \eta .
$$

One may write

$$
\begin{aligned}
|S(\xi, \tau)| \leqq \sum_{n=1}^{\infty} \mid & \frac{\varphi_{n}(\xi)}{\lambda_{n}} \int_{a}^{b} \varphi_{n}(\eta) u_{0}(\eta) d \eta|| \tau-\tau_{0} \mid \\
& \times\left|1+\frac{1}{2 !}\left(\frac{\tau-\tau_{0}}{\lambda_{n}}\right)+\frac{1}{3 !}\left(\frac{\tau-\tau_{0}}{\lambda_{n}}\right)^{2}+\cdots\right|
\end{aligned}
$$

Hence, since the $\lambda_{n}$ are bounded from zero, i.e., $\left|\lambda_{n}\right|>\alpha>0$ for all $n$, it follows that

$$
\left|\tau-\tau_{0}\right|\left|1+\frac{1}{2 !}\left(\frac{\tau-\tau_{0}}{\lambda_{n}}\right)+\frac{1}{3 !}\left(\frac{\tau-\tau_{0}}{\lambda_{n}}\right)^{2}+\cdots\right|
$$

has an upper bound $M$ for all $\tau$ 's for which $\left|\tau-\tau_{0}\right| \leqq c$. Furthermore, the infinite series

$$
\sum_{n=1}^{\infty} \frac{\varphi_{n}(\xi)}{\lambda_{n}} \int_{a}^{b} \varphi_{n}(\eta) u_{0}(\eta) d \eta
$$

converges absolutely uniformly in the interval $a \leqq \xi \leqq b$, by

${ }^{*}$ G. Vivanti, Elementi della Teoria delle Equazioni integrali, $\S 61$, $\S 71, \S 76$. 
the Hilbert-Schmidt expansion theorem.* Thus, the series representing $S(\xi, \tau)$ converges uniformly for $a \leqq \xi \leqq b$, $\left|\tau-\tau_{0}\right| \leqq c$, so that $S(\xi, \tau)$ is a continuous function for all $(\xi, \tau)$ lying in this same region.

Differentiating $S(\xi, \tau)$ with respect to $\tau$, one obtains

$$
\sum_{n=1}^{\infty} \frac{\varphi_{n}(\xi)}{\lambda_{n}} e^{\left(\tau-\tau_{0}\right) / \lambda_{n}} \int_{a}^{b} \varphi_{n}(\eta) u_{0}(\eta) d \eta,
$$

which is dominated by*

$$
e^{\left|\tau-\tau_{0}\right| / a} \int_{a}^{b} K(\xi, \eta) u_{0}(\eta) d \eta,
$$

from which it follows that $v(\xi, \tau)$ has a derivative which converges uniformly for all $\tau$ 's of $\left|\tau-\tau_{0}\right| \leqq c$.

Substituting (3) in (1), one finds

$$
\begin{aligned}
& \sum_{n=1}^{\infty} \frac{\varphi_{n}(\xi)}{\lambda_{n}} e^{\left(\tau-\tau_{0}\right) / \lambda_{n}} \int_{a}^{b} \varphi_{n}(\eta) u_{0}(\eta) d \eta=\int_{a}^{b} K(\xi, \eta) u_{0}(\eta) d \eta \\
& \quad+\int_{a}^{b} K(\xi, \eta) \sum_{n=1}^{\infty} \varphi_{n}(\eta) d \eta \int_{a}^{b} \varphi_{n}(\zeta) u_{0}(\zeta) d \zeta\left(e^{\left(\tau-\tau_{0}\right) / \lambda_{n}}-1\right) .
\end{aligned}
$$

Since the second term on the right-hand side converges uniformly, one may integrate term by term, after which the right hand side reduces to

$$
\begin{array}{r}
\int_{a}^{b} K(\xi, \eta) u_{0}(\eta) d \eta+\sum_{n=1}^{\infty} \frac{\varphi_{n}(\xi)}{\lambda_{n}} e^{\left(\tau-\tau_{0}\right) / \lambda_{n}} \int_{a}^{b} \varphi_{n}(\eta) u_{0}(\eta) d \eta \\
-\sum_{n=1}^{\infty} \frac{\varphi_{n}(\xi)}{\lambda_{n}} \int_{a}^{b} \varphi_{n}(\eta) u_{0}(\eta) d \eta
\end{array}
$$

which is simply the left-hand side of (4), by the HilbertSchmidt theorem. Hence, (3) is a solution of (1).

The uniqueness of the solution may be shown as follows: Equation (1) with the initial condition is evidently equivalent to the integral equation

$$
u(\xi, \tau)=\int_{\tau_{0}}^{\tau} \int_{a}^{b} K(\xi, \eta) u(\eta, \tau) d \eta d \tau+u_{0}(\xi) .
$$

Suppose there could be another solution $\bar{u}(\xi, \tau)$ with the same

*Vivanti, $\$ 89$. 
properties as $u(\xi, \tau)$. Then

Hence,

$$
\bar{u}(\xi, \tau)=\int_{\tau_{0}}^{\tau} \int_{a}^{b} K(\xi, \eta) \bar{u}(\eta, \tau) d \eta d \tau+u_{0}(\xi)
$$

$$
\bar{u}(\xi, \tau)-u(\xi, \tau)=\int_{\tau_{0}}^{\tau} \int_{a}^{b} K(\xi, \eta)[\bar{u}(\eta, \tau)-u(\eta, \tau)] d \eta d \tau,
$$

and calling $U$ the maximum of $|\bar{u}(\eta, \tau)-u(\eta, \tau)|$ and $K$ the maximum of $K(\xi, \eta)$, in their respective regions of definitions, one may write

$$
|\bar{u}(\xi, \tau)-u(\xi, \tau)| \leqq K U\left|\tau-\tau_{0}\right||b-a|,
$$

and by continued application of this inequality, one finally arrives at the relation

$$
|\bar{u}(\xi, \tau)-u(\xi, \tau)| \leqq[K U(b-a)]^{n} \frac{\left|\tau-\tau_{0}\right|^{n}}{n !},
$$

from which, by a passage to the limit as $n \rightarrow \infty$, one obtains the desired result.

CoRollary 1. If the initial function $u_{0}(\xi)$ has the form $\int_{a}^{b} K(\xi, \eta) \rho(\eta) d \eta$, where $\rho(\eta)$ is real and continuous in $a \leqq \eta$ $\leqq b$, then the solution (3) may be put in the form

$$
\begin{aligned}
v(\xi, \tau) & =\sum_{n=1}^{\infty} \frac{\varphi_{n}(\xi)}{\lambda_{n}} e^{\left(\tau-\tau_{0}\right) / \lambda_{n}} \int_{a}^{b} \varphi_{n}(\eta) \rho(\eta) d \eta \\
& =\sum_{n=1}^{\infty} \varphi_{n}(\xi) e^{\left(\tau-\tau_{0}\right) / \lambda_{n}} \int_{a}^{b} \varphi_{n}(\eta) u_{0}(\eta) d \eta .
\end{aligned}
$$

This follows immediately upon substitution of $\int_{a}^{b} K(\xi, \eta) \rho(\eta) d \eta$ for $u_{0}(\xi)$ in (3) and an application of the Hilbert-Schmidt expansion.

Corollary 2. The series in the solution (3) will terminate, i.e., will have the form

$$
u_{0}(\xi)+\sum_{n=1}^{m} \varphi_{n}(\xi)\left(e^{\left(\tau-\tau_{0}\right) / \lambda_{n}}-1\right) \int_{a}^{b} \varphi_{n}(\eta) u_{0}(\eta) d \eta,
$$

when, and only when, the kernel $K$ can be written in the form

$$
K(\xi, \eta)=\sum_{n=1}^{m} \frac{\varphi_{n}(\xi) \varphi_{n}(\eta)}{\lambda_{n}} .
$$


It is understood, of course, that corresponding to each root of multiplicity $p$ there will be $p$ terms in (6).

This is an immediate consequence of a well-known result in the theory of integral equations. * For example, the solution of the integro-differential equation

$$
\frac{\partial}{\partial \tau} u(\xi, \tau)=\int_{0}^{\pi / 2} \xi \eta u(\eta, \tau) d \eta
$$

passing through $\tau_{0}$ and $u_{0}(\xi)=\int_{0}^{\pi / 2} \xi \eta \sin \eta d \eta=\xi$ is given by $\xi e^{\left.\pi^{3(\tau-\tau}\right) / 24}$, since the only normed characteristic function is $\frac{2}{\pi} \sqrt{\frac{6}{\pi}} \xi$ with the characteristic number $24 / \pi^{3}$.

\section{§ 2. The Skew-Symmetric Kernel.}

If it is now supposed that $K(\xi, \eta)=-K(\eta, \xi)$, the corresponding theorem is as follows:

Theorem. Through the element $\left(\tau_{0}, u_{0}\right)$ there exists one and but one solution

$$
v(\xi, \tau)=u_{0}(\xi)+\frac{1}{2} \sum_{n=1}^{\infty}\left[\bar{d}_{n} \varphi_{n}(\xi)\left(e^{\left(\tau-\tau_{0}\right) / \lambda_{n}}-1\right)\right.
$$

$$
\left.+d_{n} \bar{\varphi}_{n}(\xi)\left(e^{\left(\tau-\tau_{0}\right) / \bar{\lambda}_{n}}-1\right)\right]
$$

reducing for $\tau=\tau_{0}$ to $u_{0}(\xi)$, where

$$
d_{n}=\int_{a}^{b} \varphi_{n}(\eta) u_{0}(\eta) d \eta
$$

and where $\bar{d}_{n}, \bar{\lambda}_{n}$, and $\bar{\varphi}_{n}(\xi)$ are the conjugates of $d_{n}, \lambda_{n}$, and $\varphi_{n}(\xi)$ respectively.

Here the $\lambda_{n}, \bar{\lambda}_{n}$ are the characteristic numbers and the $\varphi_{n}(\xi)$, $\bar{\varphi}_{n}(\xi)$ are the characteristic functions which are supposed normed and biorthogonalized. The proof of this theorem is like that of the preceding one and will not be given. It suffices to remark that in this case use is made of a development analogous to that of Hilbert-Schmidt for the symmetric case. $\dagger$

* Lalesco, Introduction à la Théorie des Equations intégrales (1912), p. 66 .

$\dagger$ Lalesco, p. 77. 
By making use of the particular form of the characteristic numbers, one may put (7) into a somewhat different form. It is well-known that the characteristic numbers of a skewsymmetric kernel are of the form $\pm \nu i$ and that if $\nu i$ is a characteristic number with $\varphi(\xi)$ as characteristic function, then $-\nu i$ is also a characteristic number with $\bar{\varphi}(\xi)$ as corresponding characteristic function. If in (7) one introduces the notations

$$
\begin{aligned}
\lambda_{n} & =\nu_{n} i, & \varphi_{n}(\xi) & =\psi_{n}(\xi)+i \chi_{n}(\xi), \\
e_{n} & =\int_{a}^{b} \psi_{n}(\eta) u_{0}(\eta) d \eta, & f_{n} & =\int_{a}^{b} \chi_{n}(\eta) u_{0}(\eta) d \eta,
\end{aligned}
$$

one finds, after some reductions, that it may be written

$$
\begin{aligned}
v(\xi, \tau)=u_{0}(\xi)+\sum_{n=1}^{\infty}[ & \left\{e_{n} \psi_{n}(\xi)+f_{n} \chi_{n}(\xi)\right\}\left(\cos \frac{\tau-\tau_{0}}{\nu_{n}}-1\right) \\
& \left.+\left\{e_{n} \chi_{n}(\xi)-f_{n} \chi_{n}(\xi)\right\} \sin \frac{\tau-\tau_{0}}{\nu_{n}}\right] .
\end{aligned}
$$

Just as in the symmetric case, it is to be remarked here that when $u_{0}(\xi)$ has the special form $\int_{a}^{b} K(\xi, \eta) \rho(\eta) d \eta$, where $\rho(\eta)$ is a real continuous function, then (7) reduces to

$$
v(\xi, \tau)=\sum_{n=1}^{\infty}\left[\bar{g}_{n} \frac{\varphi_{n}(\xi)}{\lambda_{n}} e^{\left(\tau-\tau_{0}\right) / \lambda_{n}}+g_{n} \frac{\bar{\varphi}_{n}(\xi)}{\bar{\lambda}_{n}} e^{\left(\tau-\tau_{0}\right) / \bar{\lambda}_{n}}\right],
$$

where

$$
g_{n}=\int_{a}^{b} \varphi_{n}(\xi) \rho(\eta) d \eta
$$

and $\bar{g}_{n}$ is the conjugate of $g$. Moreover, (8) becomes

$$
v(\xi, \tau)=\sum_{n=1}^{\infty} \frac{2}{\nu_{n}}\left[\beta_{n}(\xi) \cos \frac{\tau-\tau_{0}}{\nu_{n}}+\gamma_{n}(\xi) \sin \frac{\tau-\tau_{0}}{\nu_{n}}\right],
$$

where

$$
\begin{aligned}
\beta_{n}(\xi) & =h_{n} \chi_{n}(\xi)-k_{n} \psi_{n}(\xi), & \gamma_{n}(\xi) & =h_{n} \psi_{n}(\xi)+k_{n} \chi_{n}(\xi), \\
h_{n} & =\int_{a}^{b} \psi_{n}(\xi) \rho(\xi) d \xi, & k_{n} & =\int_{a}^{b} \chi_{n}(\xi) \rho(\xi) d \xi .
\end{aligned}
$$

Finally, in view of the fact* that every skew-symmetric kernel

\footnotetext{
${ }^{*}$ Lalesco, p. 77.
} 
having a finite number of characteristic numbers is necessarily of the form

$$
\sum_{n=1}^{m}\left(\frac{\varphi_{n}(\xi) \bar{\varphi}_{n}(\eta)}{\nu_{n} i}+\frac{\bar{\varphi}_{n}(\xi) \varphi_{n}(\eta)}{-\nu_{n} i}\right),
$$

it follows that for such kernels and only for such, the solution (7) has a finite number of terms.

As an example of an integro-differential equation of this last type, consider the equation

$$
\frac{\partial}{\partial \tau} u(\xi, \tau)=\int_{0}^{2 \pi} \sin \sigma(\xi-\eta) u(\eta, \tau) d \eta
$$

where $\sigma$ is an odd integer. Now, since

$$
\sin \sigma(\xi-\eta)=\frac{\frac{e^{\sigma i \xi}}{\sqrt{2 \pi}} \frac{e^{-\sigma i \eta}}{\sqrt{2 \pi}}}{i / \pi}+\frac{\frac{e^{-\sigma i \xi}}{\sqrt{2 \pi}} \frac{e^{\sigma i \eta}}{\sqrt{2 \pi}}}{-i / \pi},
$$

it is clear that the only characteristic numbers of the kernel $\sin \sigma(\xi-\eta)$ are $i / \pi$ and $-i / \pi$ with the corresponding normed biorthogonal characteristic functions $e^{\sigma i \xi} / \sqrt{2 \pi}, e^{-\sigma i \xi} / \sqrt{2 \pi}$. Hence, the solution of (11), which reduces for $\tau=\tau_{0}$ to an arbitrary continuous function $u_{0}(\xi)$, is, according to (7)

$$
\begin{array}{r}
v(\xi, \tau)=u_{0}(\xi)+\frac{1}{2 \pi}\left[e^{-\sigma i \xi}\left(e^{-\pi i\left(\tau-\tau_{0}\right)}-1\right)\right] \int_{0}^{2 \pi} e^{\sigma i \eta} u_{0}(\eta) d \eta \\
+e^{\sigma i \xi}\left(e^{\pi i\left(\tau-\tau_{0}\right)}-1\right) \int_{0}^{2 \pi} e^{-\sigma i \eta} u_{0}(\eta) d \eta
\end{array}
$$

or, by formula (8)

$$
\begin{aligned}
v(\xi, \tau)=u_{0}(\xi)-\frac{2}{\pi} \int_{0}^{2 \pi} \sin \frac{\pi\left(\tau-\tau_{0}\right)}{2} \sin & {\left[\frac{\pi\left(\tau-\tau_{0}\right)}{2}\right.} \\
& -\sigma(\xi-\eta)] u_{0}(\eta) d \eta
\end{aligned}
$$

§3. Some Extensions and Applications of the Preceding Results.

In recalling the proofs of formulas (3) and (7), one sees that the essential feature was the fact that the symmetric and skew-symmetric kernels had an expansion (HilbertSchmidt) associated with them. This is also true for the symmetrizable kernels (Marty). A kernel $K(\xi, \eta)$ is said 
to be symmetrizable if there exists a definite symmetric kernel $G(\xi, \eta)$ such that either

or

$$
P(\xi, \eta)=\int_{a}^{b} G(\xi, \zeta) K(\zeta, \eta) d \zeta
$$

$$
Q(\xi, \eta)=\int_{a}^{b} K(\xi, \zeta) G(\zeta, \eta) d \zeta
$$

is symmetric.* As in the symmetric case, the characteristic numbers $\lambda_{n}$ are real and the theorem on the development says that, if $f(\xi)$ is of the form $\int_{a}^{b} K(\xi, \eta) h(\eta) d \eta$, then $\dagger$

$$
f(\xi)=\sum_{n=1}^{\infty} \frac{\iint G(\eta, \zeta) \varphi_{n}(\zeta) f(\eta) d \eta d \zeta}{\lambda_{n}} \varphi_{n}(\xi)
$$

where the $\varphi_{n}(\xi)$ are the characteristic functions of $K(\xi, \eta) . \ddagger$ One could then easily write down the form of the solution corresponding to (3). It is to be remarked that both the symmetric and skew-symmetric kernels are special instances of symmetrizable kernels, as well as the kernels $A(\xi) G(\xi, \eta)$, $A(\xi) G(\xi, \eta) B(\eta)$ and $\int_{a}^{b} H(\xi, \eta) G(\zeta, \eta) d \zeta$, where $G$ is a definite symmetric kernel and $H$ is symmetric.

One could also generalize farther by considering equations of the type

$$
\frac{\partial}{\partial \tau} u(p, \tau)=J_{q r} K(p, q) u(r, \tau),
$$

where $p, q$, and $r$ have arbitrary ranges $\$ ; ~ K$ is hermitian, i.e., $\overline{K(p, q)}=K(q, p)$; and $J$ is a linear functional operation. In view of Moore's generalization of the Hilbert-Schmidt theory, one could easily generalize the results of Section 1.

It would be interesting to see how to express the solution of the integro-differential equation (1) in terms of the characteristic functions and numbers when the kernel is perfectly general, as in Volterra's case. For this purpose it would

* Lalesco, p. 78.

† Lalesco, p. 84.

$\ddagger$ The functions $\varphi_{n}(\xi)$ and $\psi_{n}(\xi)=\int G(\xi, \eta) \varphi_{n}(\eta) d \eta$ are supposed normed and biorthogonalized.

$\S \mathrm{E}$. H. Moore, "On the foundations of the theory of linear integral equations," BULlETiN, vol. 18 (1912), pp. 334-362. Hildebrandt, "On a theory of linear differential equations in general analysis," Trausactious Amer. Math. Society, vol. 18 (1917), pp. 73-96. 
seem that one would have to consider the elementary divisors of the Fredholm determinant, as in the theory of the finite system of differential equations with constant coefficients.

Before leaving this subject, one should notice that the integro-differential equation

$$
\frac{\partial}{\partial \tau} u(\xi, \tau)=y(\tau) u(\xi, \tau)+\int_{a}^{b} K(\xi, \eta) u(\eta, \tau) d \eta,
$$

where $y(\tau)$ is a continuous function of $\tau$ in the interval $\left|\tau-\tau_{0}\right| \leqq c$ and $K(\xi, \eta)$ is, for example, symmetric, is readily reducible to an equation of type (1). For, multiplying both sides of (12) by $e^{\int_{\tau_{0}}^{\tau} y(\tau) d \tau}$ and replacing $e^{\int_{\tau_{0}}^{\tau} y(\tau) d \tau} u(\xi, \eta)$ by $w(\xi, \tau)$, one finds the equation

$$
\frac{\partial}{\partial \tau} w(\xi, \tau)=\int_{a}^{b} K(\xi, \eta) w(\eta, \tau) d \eta,
$$

so that the solution of (12), having an initial function $u_{0}(\xi)$, is given by

$$
\begin{aligned}
& v(\xi, \tau)=u_{0}(\xi) e^{-\int_{\tau_{0}}^{\tau} y(\tau) d \tau}+\sum_{n=1}^{\infty} \varphi_{n}(\xi)\left(e^{\left(\tau-\tau_{0}\right) / \lambda_{n}}-1\right) \\
& \times e^{-\int_{\tau_{0}}^{\tau} y(\tau) d \tau} \int_{a}^{b} \varphi_{n}(\eta) u_{0}(\eta) d \eta .
\end{aligned}
$$

In particular, if $y(\tau) \equiv 1$, this becomes

$$
\begin{aligned}
v(\xi, \tau) & =e^{\tau_{0}-\tau} u_{0}(\xi) \\
+ & \sum_{n=1}^{\infty} \varphi_{n}(\xi)\left(e^{\left(\tau-\tau_{0}\right)\left(1-\lambda_{n}\right) / \lambda_{n}}-e^{\tau_{0}-\tau}\right) \int_{a}^{\circ b} \varphi_{n}(\eta) u_{0}(\eta) d \eta .
\end{aligned}
$$

Consider also the non-homogeneous equation

$$
\frac{\partial}{\partial \tau} u(\xi, \tau)=\int_{a}^{b} K(\xi, \eta) u(\eta, \tau) d \eta+z(\xi),
$$

where $z(\xi)$ is continuous and has the form $\int_{a}^{b} K(\xi, \eta) x(\eta) d \eta$. Then it can be readily verified that the unique solution passing through $u_{0}(\xi)$ is given by

$$
\begin{aligned}
& v(\xi, \tau)=u_{0}(\xi)+\sum_{n=1}^{\infty} \varphi_{n}(\xi)\left(e^{\left(\tau-\tau_{0}\right) / \lambda_{n}}-1\right) \\
& \quad \int_{a}^{b} \varphi_{n}(\eta)\left[u_{0}(\eta)+z(\eta)\right] d \eta,
\end{aligned}
$$

which obviously reduces to (3) when $z(\eta)=0$. 
It is now desired to point out an analogy between integrodifferential equations and partial differential equations. Consider the problem of the free vibrations of an elastic string. Let the string when stretched be of length unity and of homogeneous density. Moreover, let the initial position of the string be $u_{0}(\xi)$ and let each particle start its motion with the initial velocity zero. Then, it can be shown that, if the units are chosen properly, the differential equation of the problem will be given by*

$$
\begin{aligned}
\frac{\partial^{2}}{\partial \tau^{2}} u(\xi, \tau) & =c^{2} \frac{\partial^{2}}{\partial \xi^{2}} u(\xi, \tau), \quad(c=\text { constant }), \\
u\left(\xi, \tau_{0}\right) & =u_{0}(\xi), \quad u(0, \tau)=u(1, \tau)=0 .
\end{aligned}
$$

By following the usual methods for solving such systems, one obtains that a solution of (17) is given by

(18) $u(x, \tau)=2 \sum_{n=1}^{\infty} \sin n \pi x e^{n^{2} \pi^{2}\left(\tau-\tau_{0}\right)} \int_{0}^{1} u_{0}(\xi) \sin n \pi \xi d \xi$,

if it is assumed that $u_{0}(\xi)$ is of such a character that it is expansible in a Fourier series.

Now consider the integro-differential equation

$$
\frac{\partial}{\partial \tau} u(\xi, \tau)=\int_{0}^{1} K(\xi, \eta) u(\eta) d \eta, \quad u\left(\xi, \tau_{0}\right)=u_{0}(\xi),
$$

where

$$
K(\xi, \eta)= \begin{cases}(1-\eta) \xi, & 0 \leqq \xi \leqq \eta \\ \eta(1-\xi), & \eta \leqq \xi \leqq 1\end{cases}
$$

is the Green's function relative to the system (17).

It is well known that the characteristic numbers of this kernel have the form $n^{2} \pi^{2}$ with the normed orthogonal characteristic functions $\sqrt{2} \sin n \pi \xi$. So that, if one supposes that $u_{0}(\xi)$ has the form $\int_{0}^{1} K(\xi, \eta) \rho(\eta) d \eta$ and hence is expansible in a Fourier series, one obtains immediately from (5) that the general solution of (19) is given by

(20) $u(x, \tau)=2 \sum_{n=1}^{\infty} \sin n \pi x e^{\left(\tau-\tau_{0}\right) / n^{2} \pi^{2}} \int_{0}^{1} u_{0}(\xi) \sin n \pi \xi d \xi$, $\S 83$.

* Weber-Riemann, Lehrbuch der partiellen Differential-Gleichungen, 
which is seen to agree with (18), except for the fact that the coefficient of $\tau-\tau_{0}$ in the exponent of $e$ in (20) is the reciprocal of that in (19). This brings out the relation between systems (19) and (17). This is readily generalized to all systems like (17) which lead to self-adjoint differential equations of the second order with boundary conditions.

Harvard UNIVERSity, October 21, 1919.

\section{ON A PENCIL OF NODAL CUBICS.}

BY PROFESSOR NATHAN ALTSHILLER-COURT.

(Read before the American Mathematical Society December 31, 1919.)

Consider a pencil $\Gamma$ of nodal cubics having in common three collinear points, the double point, and the two tangents at this point.

1. Let $\Gamma_{n}$ be one of the cubics of the pencil $\Gamma$. A variable secant passing through one of the three basic collinear points $A, B, C$, say $A$, cuts $\Gamma_{n}$ in pairs of points which are projected from the double point $O$ by an involution of rays, the two tangents $O T_{1}, O T_{2}\left(T_{1}, T_{2}\right.$ are points of the basic line $\left.A B C\right)$ to $\Gamma_{n}$ at $O$ being a pair of conjugate elements in this involution. $^{1}$ The lines $O B, O C$, are obviously another pair of conjugate elements in this involution. The double lines $O M_{n}$, $O M_{n}{ }^{\prime}$, of this involution project from $O$ the two points of contact $M_{n}, M_{n}^{\prime}$ of the two tangents from $A$ to $\Gamma_{n}$.

When $\Gamma_{n}$ describes the pencil $\Gamma$ the two pairs of lines $O T_{1}$, $O T_{2}$ and $O B, O C$, remain fixed, by hypothesis, hence the involution

$$
O\left(T_{1} T_{2}, B C, M_{n} M_{n}, M_{n}{ }^{\prime} M_{n}{ }^{\prime}\right)
$$

is fixed, and therefore also its doubles lines $O M_{n}, O M_{n}^{\prime}$. Consequently: The points of contact of the pairs of tangents from one of the basic points to curves of the pencil $\Gamma$ lie on two fixed lines passing through the double point.

2. The corresponding point $A_{n}^{\prime}$ of $A$ on $\Gamma_{n}$ is projected

${ }^{1}$ M. Chasles, "Mémoire sur la construction des racines des équations du troisième et du quatrième degré," Comptes Rendus de l'Académie des Sciences, tome 41, p. 677. E. de Jonquières, Mélanges de Géométrie pure, 1856, p. 180. 